\title{
Prognostic significance of acute presentation with emergency complications of gastric cancer
}

\author{
Guy R.J.C. Blackshaw ${ }^{1}$, Michael R. Stephens ${ }^{1}$, Wyn G. Lewis ${ }^{1}$, Holly J. Paris ${ }^{1}$, Jonathan D. Barky ${ }^{1}$, \\ Paul Edwards ${ }^{1}$, and Miles C. Allison ${ }^{2}$ \\ ${ }^{1}$ Department of Surgery, Royal Gwent Hospital, Newport, NP20 2UB, UK \\ ${ }^{2}$ Department of Gastroenterology, Royal Gwent Hospital, Newport, UK
}

\begin{abstract}
Background. Although acute complications necessitating emergency hospital admission are well documented in patients with carcinoma of the colon, comparable data for patients with gastric carcinoma is thin. The aim of this study, therefore, was to examine the outcomes of patients presenting to hospital as acute admissions with emergency complications of previously undiagnosed gastric cancer.

Methods. Three hundred consecutive patients with gastric adenocarcinoma were studied prospectively, and subdivided into two groups according to whether the patients were referred as acute emergencies $(n=116)$ or as outpatients $(n=$ 184).

Resuslts. The commonest emergency complications were: abdominal pain (57\%), vomiting (41\%), gastrointestinal bleeding $(37 \%)$, dysphagia (26\%), and a palpable mass $(18 \%)$. Stages of disease were significantly more advanced in patients presenting acutely (I:II:III:IV = 7:11:27:71) compared with patients referred via outpatients $\left(20: 23: 50: 91, \chi^{2}=\right.$ 3.955; DF, $1 ; P=0.047)$. R0 gastrectomy was significantly less likely after acute presentation (23 patients; $20 \%$ ) compared with patients referred via outpatients (70 patients; $38 \% ; \chi^{2}=$ 11.037; DF, 1; $P=0.001)$. Cumulative 5-year survival for patients referred acutely was $9 \%$, compared with $22 \%$ after outpatient referral $\left(\chi^{2}=9.11 ; \mathrm{DF}, 1 ; \boldsymbol{P}=\mathbf{0 . 0 0 2 5}\right)$. Multivariate analysis revealed two factors to be significantly and independently associated with durations of survival: stage of disease (hazard ratio [HR], 1.742; 95\% confidence interval [CI], 1.493-2.034; $P=0.0001)$ and presentation with acute complications (HR, 1.561; 95\% CI, 1.151-2.117; $P=0.004)$.

Conclusion. Emergency complications of gastric cancer are a significant and independent prognostic marker of poor outcome.
\end{abstract}

Offprint requests to: W.G. Lewis

Presented at: British Society of Gastroenterology, Birmingham 2003, and 5th International Gastric Cancer Congress, Rome 2003.

Received: December 4, 2003 / Accepted: February 3, 2004
Key words Emergency admission · Gastric cancer · Surgery

\section{Introduction}

Gastric cancer is now the second most common cancer worldwide, accounting for 10000 new diagnoses and 7500 deaths each year in the United Kingdom alone $[1,2]$. The stage of disease at presentation is the most important prognostic factor, but delays in diagnosis remain common [3], and despite the introduction of open access gastroscopy, as many as one in three patients in Britain continue to present with advanced disease that is incurable by means of surgery [4].

Acute complications necessitating emergency hospital admission are well documented in patients with carcinoma of the colon [5]. Moreover, outcomes of treatment and durations of survival are poorer in these patients when compared with patients diagnosed via conventional methods [6]. Although there are recent large and detailed audit reports regarding esophagogastric cancer from the United Kingdom regions with the highest prevalence of gastric cancer from both Wales and Scotland [7,8], as far as we are aware, there are no reports in the literature regarding the outcomes of patients with previously undiagnosed gastric cancers who develop emergency complications of their tumors, necessitating acute hospital admission and treatment.

The aims of this study, therefore, were to quantify the proportion of patients admitted to a large District General Hospital in the United Kingdom with emergency complications of previously undiagnosed gastric cancer; to identify factors contributing to possible delays in diagnosis; to determine the stages of disease at presentation; to audit outcomes of treatments; and to determine long-term survival. It was our hypothesis that gastric 
cancer is associated with an especially poor prognosis when presenting as an emergency with acute complications.

\section{Patients and methods}

Between October 1, 1995 and October 31, 2003, 300 consecutive patients with adenocarcinoma of the stomach presented to the Royal Gwent Hospital. Their median age was 71 years (range, 27-93 years), with 17 patients aged less than 50 years. The demographic details of the patients are shown in Table 1. The overall diagnostic delay in weeks was recorded for each patient. Dates were recorded according to the patient's recollection and were cross-referenced with the case notes. Indices of multiple deprivation (IMD) were obtained from the Office for National Statistics [9]. The time to diagnosis was measured from the date when the patient first experienced significant symptoms to the establishment of a histological diagnosis of malignancy. All patients underwent gastroscopy and staging investigations (chest X-ray and computed tomography [CT]), together with preoperative laparoscopy if surgery was contemplated (131 patients). Ethical approval was granted by the Gwent Research Ethics Committee.

Table 1. Details of the 300 patients related to method of referral

\begin{tabular}{lcc}
\hline & Acute & Outpatient \\
\hline Number & 116 & 184 \\
Age, years (range) & $74(27-93)$ & $70(35-90)^{\mathrm{a}}$ \\
Sex (m:f) & $72: 44$ & $126: 58$ \\
Anemia & $62(54)$ & $33(18)^{*}$ \\
Hematemesis/melena & $43(37)$ & $19(10)^{*}$ \\
Mass & $21(18)$ & $16(9)^{* *}$ \\
Vomiting & $48(41)$ & $58(32)^{* *}$ \\
Anorexia & $35(30)$ & $36(20)^{* *}$ \\
Weight loss & $43(37)$ & $93(50)^{* *}$ \\
Dyspepsia & $70(60)$ & $142(77)^{* *}$ \\
Dysphagia & $30(26)$ & $30(16)^{* *}$ \\
Abdominal pain & $66(57)$ & $99(54)$ \\
Site Cardia & $34(29)$ & $76(41)$ \\
$\quad$ Body & $15(13)$ & $30(16)$ \\
$\quad$ Antrum & $35(30)$ & $51(28)$ \\
$\quad$ Linitis plastica & $32(28)$ & $29(16)$ \\
Median tumor size (cm) & $6(3-12)$ & $4.5(2-13)$ \\
Stage I & $7(6)$ & $20(11)$ \\
Stage II & $11(10)$ & $23(13)$ \\
Stage III & $27(23)$ & $50(27)$ \\
Stage IV & $71(61)$ & $91(49)^{* *}$ \\
ASA grade (I:II:III:IV) & $4: 27: 27: 58$ & $13: 60: 85: 26^{*}$ \\
& &
\end{tabular}

$* P=0.0001 ; * * P<0.05$

Figures are numbers of patients $(\%)$

ASA, American Society of Anesthesiologists

${ }^{a}$ Median

\section{Surgical treatment}

All of the surgery was performed by a single surgeon (W.G.L.) with a special interest in esophagogastric cancer. It is our policy to perform a modified radical D2 resection with extended lymphadenectomy, but preserving the pancreas and spleen where possible, as described originally by the Leeds group and more recently by ourselves $[10,11]$. The definition of potentially curative resection was that all visible tumor was removed and that both proximal and distal resection margins were free of tumor on histological examination. All tumors were initially staged in accordance with the 1987 Unified TNM classification of gastric cancer [12], and thereafter with the 1997 Unified TNM classification [13]. No patients underwent neoadjuvant chemotherapy prior to surgery.

\section{Follow up}

Patients undergoing surgery were reviewed every 3 months for the first year and every 6 months thereafter. Endoscopy and CT were arranged if recurrent disease was suspected. Causes of death were sought from case notes, pathology records, and general practitioners' records. None of the 300 patients were lost to follow up and 244 patients $(81 \%)$ were followed up for a minimum of 5 years or until death.

\section{Statistical analysis}

Statistical analysis appropriate for non-parametric data was used [14]. Grouped data were expressed as median (interquartile range). Cumulative survival was calculated by the life table method of Kaplan and Meier [15]. Differences in survival times between groups of patients were analyzed by the log rank method [16]. Cox's proportional hazards model was used to assess the prognostic value of individual variables. Data analysis was carried out with the Statistical Package for Social Sciences (SPSS) version 11.5 (SPSS, Chicago, IL, USA).

\section{Results}

Of the 300 patients, 116 were referred as emergencies with acute complications of their tumors. The remaining 184 patients were referred as outpatients, either by conventional outpatient clinic letter (99 patients) or via the open access endoscopy service (85 patients).

\section{Symptoms at presentation (Table 1)}

Anemia $(P<0.001)$, hematemesis $(P<0.001)$, mass $(P=0.02)$, weight loss $(P=0.02)$, and dysphagia 
$(P=0.04)$ were significantly more common in patients admitted acutely when compared with those referred as outpatients. Dysphagia was significantly more common in patients with tumors of the cardia when compared to more distal tumors $(P=0.034)$.

\section{Stages of disease at presentation (Table 1)}

Stages of disease were significantly more advanced in patients presenting acutely, compared with patients referred as outpatients, with $61 \%$ having advanced metastatic disease at presentation $\left(\chi^{2}=3.955\right.$; DF, 1 ; $P=0.047)$.

\section{Delays in diagnosis}

The median delay from the onset of symptoms to a definitive diagnosis for the 116 acute patients was 6 weeks (range, 1-129 weeks), compared with 20 weeks (range, 1-175 weeks) for patients referred via the open access gastroscopy service $(P=0.0001)$, and 20 weeks (range, 1-175 weeks) for patients referred via outpatients $(P=0.0001)$.

\section{Indices of multiple deprivation}

There was no significant difference between the deprivation scores of acute patients (median, 22.14 [range, 1.79-63.71]) when compared with the scores of outpatients (21.3 [3.29-63.71]).

\section{Blind acid suppression prior to diagnosis}

Forty-four of the 116 patients (38\%) presenting as emergencies had received blind acid suppression therapy from their general practitioners prior to investigation, compared with 55 of the 184 patients (30\%) referred via outpatient clinics $\left(\chi^{2}, 2.08 ; \mathrm{DF}, 1 ; P=\right.$ $0.149)$. There was no significant correlation between the

Table 2. Details of the surgery related to method of referral

\begin{tabular}{lcc}
\hline & Acute & Outpatient \\
\hline Operation & & \\
Subtotal gastrectomy & $14(13)$ & $39(37)$ \\
Total gastrectomy & $16(10)$ & $35(28)$ \\
Esophagogastrectomy & $1(0)$ & $8(5)$ \\
Splenectomy & 3 & 13 \\
Distal pancreatectomy & 0 & 4 \\
Transverse colectomy & 5 & 9 \\
Laparoscopy only & 12 & 36 \\
Laparotomy only & 6 & 14 \\
Bypass procedure & 4 & 3
\end{tabular}

Figures are numbers of patients; R0 resections are shown in parentheses stages of patients' tumors and whether or not patients were receiving blind acid suppression therapy.

Prior investigation of patients subsequently presenting with emergency complications

Fourteen of the patients $(12 \%)$ presenting with emergency complications had undergone prior investigations for dyspepsia within the previous 2 years. Three of these patients had undergone an endoscopy which was reported as normal, 4 patients were reported to have generalized gastritis and were awaiting repeat endoscopy, and 7 patients were under surveillance for suspicious gastric ulcers. Diagnostic delays in these patients were similar to those in the rest of the group.

\section{Details of the surgery (Table 2)}

Twenty-three of the 116 acute patients $(20 \%)$ underwent an R0 D2 gastrectomy compared with 70 of the 184 patients $(38 \%)$ referred via outpatients $\left(\chi^{2}=\right.$ 11.037 ; DF, $1 ; P=0.001)$. No patients underwent emergency surgery within $24 \mathrm{~h}$ of admission to hospital.

\section{Operative morbidity and mortality (Table 3)}

Details of the major operative morbidity and mortality are shown in Table 3. Operative morbidity was $35 \%$ in acute patients compared with $23 \%$ in patients referred via outpatient clinics $\left(\chi^{2}=2.056\right.$; DF, $\left.1 ; P=0.152\right)$. Overall operative mortality within 30 days of surgery was $14 \%$ in acute patients compared with $7 \%$ inpatients referred via outpatients $\left(\chi^{2}=1.259\right.$; DF, $\left.1 ; P=0.262\right)$. Operative mortality after potentially curative R0 gas-

Table 3. Details of the operative morbidity related to method of referral

\begin{tabular}{lcc}
\hline & Acute & Outpatient \\
\hline Anastomotic leak & $2(2)$ & $2(1)$ \\
Respiratory infection & $5(1)$ & $7(2)$ \\
Pulmonary embolus & 0 & $2(2)$ \\
Myocardial infarction & $1(1)$ & $2(2)$ \\
Arrhythmia & 0 & 1 \\
Aortic occlusion & 1 & 0 \\
CVA & 0 & 1 \\
Renal failure & 0 & 1 \\
Wound infection & 2 & 2 \\
Intraabdominal collection & 0 & 1 \\
Chylothorax & 0 & 1 \\
Catheter-related sepsis & 1 & 2 \\
DIC & $1(1)$ & 0 \\
Total & $13(5)$ & $22(7)$ \\
\hline
\end{tabular}

Figures are numbers of patients, with operative deaths in parentheses CVA, cerebro-vascular accident; DIC, disseminated intravascular coagulation 
trectomy was $4 \%$ in acute patients compared with $7 \%$ in outpatients $(P=0.636)$.

\section{Survival}

Corrected cumulative survival by treatment, calculated by life table analysis, is shown in Fig. 1. Cumulative 5-year survival for the 116 acute patients was 9\%. In contrast, 5-year survival for the 184 patients referred via outpatients was $22 \%\left(\chi^{2}=9.11 ; \mathrm{DF}, 1 ; P=0.0025\right)$. The median survival (or time to death) after acute presentation was 6 months (range, 1-87 months) compared with 12 months (range, 1-97 months) after out-patient referral. The factors found to be significantly associated with durations of survival on univariate analysis are shown in Table 4. None of the patients presenting with a palpable mass survived for 5 years, compared with $66 \%$ of patients without a mass $(P=0.0059)$; and 5-year

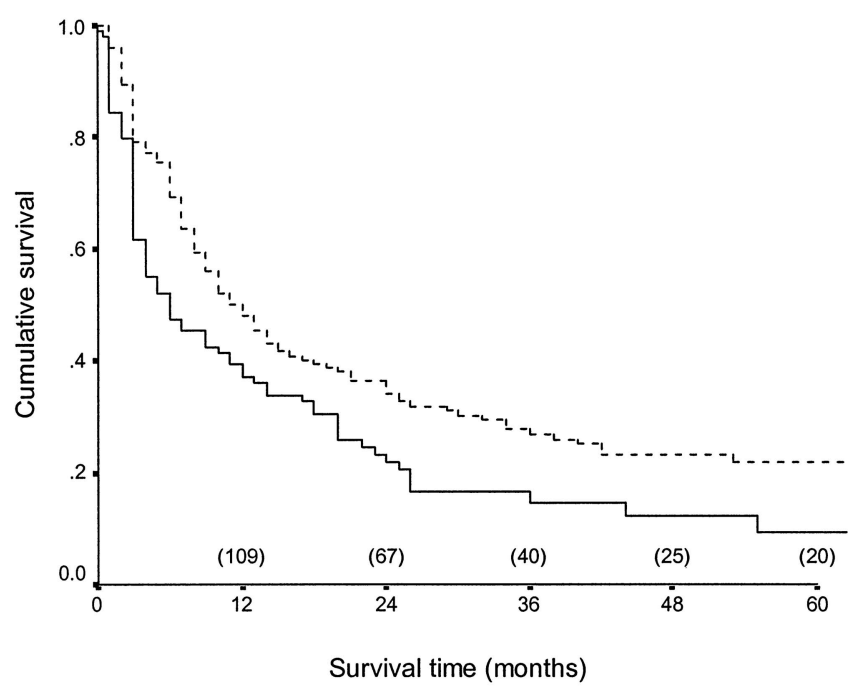

Fig. 1. Cumulative survival curves for patients with gastric cancer with respect to method of referral. $\log$ rank $\chi^{2}=9.11$; $P=0.0025$. Number of patients at risk in parentheses. Continuous line, Acute presentation; dashed line, outpatient referral

Table 4. Univariate analysis of factors associated with duration of survival

\begin{tabular}{lcl}
\hline Factor & $\chi^{2}$ & $P$ value \\
\hline Sex & 0.31 & 0.5774 \\
Lymph node ratio & 24.89 & 0.0719 \\
Acute referral & 9.25 & 0.0024 \\
Site of tumor & 22.4 & 0.00001 \\
ASA grade & 28.51 & 0.00001 \\
T stage & 33.61 & 0.00001 \\
N stage & 76.77 & 0.00001 \\
M stage & 77.69 & 0.00001 \\
Overall stage & 83.88 & 0.00001 \\
Age & 97.25 & 0.00001 \\
\hline
\end{tabular}

survival was $10 \%$ in patients presenting with anorexia compared with $80 \%$ in patients without anorexia $(P=0.0118)$.

\section{Multivariate analysis}

The prognostic variables entered into the model were age, sex, stage of the tumors, location of the tumors, type of gastrectomy (subtotal or total), and presentation with acute complications. Forward and backward stepwise regression was used. Stage of disease (HR, 1.742 ; 95\% CI, 1.493-2.034; $P=0.0001)$ and acute presentation (HR, 1.561; 95\% CI, 1.151-2.117; $P=0.004)$ were found to be the most important predictors of survival as determined by Cox's proportional hazards model. The global $\chi^{2}$ for the model was 62.537; DF, 6; $P<0.0001$.

\section{Discussion}

Patients with cancer frequently require emergency admission to hospital with complications of their tumors [5]. Indeed, the prevalence of emergency admissions to hospitals from complications of colorectal cancer was recently described by Mella et al. [5] in a large United Kingdom audit of 3221 patients from the regions of Trent and Wales. In this study no fewer than $17.1 \%$ of patients developed complications of their cancers necessitating hospital admission, and $21 \%$ of these patients had advanced incurable disease with evidence of distant metastases. The principal findings of our study were that more than one in three of a consecutive series of 300 patients developed emergency complications of their gastric cancers necessitating acute hospital admission, and no fewer than $61 \%$ had advanced incurable disease. Only $20 \%$ of these patients subsequently underwent potentially curative surgery and their overall crude 5-year survival was $9 \%$.

Open access gastroscopy has been heralded as the key to the diagnosis of gastric cancer at an early and potentially curable stage. Indeed, Whiting et al. [17] described a $26 \%$ increase in the detection of early gastric cancer in a study from Birmingham. In Japan, where gastric cancer has a much higher incidence, screening programs are effective in the detection of tumors in their early, and curable stages. This is reflected in a 5 -year survival as high as $90 \%$ for early gastric cancer [18]. Hallissey et al. [19] have shown that the effective use of open access gastroscopy in Britain can achieve similar results, with the caveat that such outcomes depend largely on the awareness and effective use of this service by general practitioners. Nevertheless, despite the widespread introduction of open access gastroscopy in Britain, as many as one in three patients continue to 
present with advanced incurable cancers [4]. Bramble et al. [20] and Suvakovic and Bramble [21] suggested that empirical use of antisecretory medication can mask the symptoms of early gastric cancer, arguably resulting in undue diagnostic delay. Blind acid suppression therapy by general practitioners occurred in $38 \%$ of the patients presenting with emergency complications and $30 \%$ of the patients referred as outpatients. This is clearly significant, as these particular patients had obviously consulted their general practitioners previously with symptoms of dyspepsia, and none were referred for a specialist opinion or open access gastroscopy. Delays in the diagnosis of gastric cancer are well documented [3], although the spectrum of this delay during the patients' journey varies considerably. For example, delay on the part of the patients in the community prior to seeking medical attention accounted for $29 \%$ of the total delay described by Martin et al. [3] from Leeds in the United Kingdom, compared with $80 \%$ reported by Wayman et al. [22] from Newcastle. In the present study, the time taken from onset of symptoms to definitive diagnosis in patients admitted as emergencies was on average only 5 weeks - one-quarter that among patients diagnosed via outpatient referral or open access gastroscopy. Possible explanations are that either the emergency patients had a more aggressive, rapidly progressive form of cancer, than patients referred and diagnosed as outpatients, or that they were truly asymptomatic prior to their emergency admission. An equally plausible explanation may be that patients who present acutely are more likely to have ignored significant symptoms and less likely to be able to accurately recall the date of onset of symptoms. These findings deserve further investigation, maybe by examining potential causes or triggers in patients referred as emergencies, such as infection with Helicobacter pylori and the possible influence of socio-economic deprivation on patterns of infection.

Stage-directed management tailored to individual patients is clearly the way forward if the benefits of greater subspecialization and a multidisciplinary team approach are to be realized [23]. New strategies are available for the treatment of patients with advanced gastric cancer. The rationale that postoperative chemotherapy may be beneficial has been under investigation for 25 years. Although a recent metaanalysis suggested a small survival advantage with an odds ratio for death in the treated group of 0.80 (95\% CI, 0.67-0.97) there remains insufficient evidence to indicate that adjuvant chemotherapy is standard treatment and clinical trials should continue [24]. Three large-phase trials of neoadjuvant chemotherapy are underway [MRC Adjuvant Gastric Infusional Chemotherapy Trial (MAGIC), Swiss Group for Clinical Cancer Research (SAKK) 43/99, and European Organisation for Research and
Treatment of Cancer (EORTC) 40954], and preliminary results of the MAGIC trial in the United Kingdom, comparing surgery alone with ECF (epirubicin, cisplatin, and 5-fluoruracil) in 503 patients, were released most recently [25]. Neoadjuvant therapy increased the R0 resection rate significantly, from $69 \%$ to $79 \%$, and pathological examination suggested a downsizing of the tumor, without any increase in operative morbidity when compared with surgery alone. Whereas progression-free survival was improved significantly, there was no advantage in overall survival after a minimum of 1-year follow up in all patients. Postoperative chemoradiotherapy has been recently evaluated in a randomized trial involving 603 patients [26], and at a median follow up of 3.3 years demonstrated a disease-free and overall survival advantage for patients treated. Patients presenting with emergency complications clearly have a worse prognosis than control patients even after appropriate surgery, and they might be candidates for a risk-adapted and tailored multimodal approach. Such an approach, however, requires further evaluation and remains investigational.

In conclusion, the widely held assumption that acute admission to hospital with emergency complications of a gastric cancer is often a harbinger of doom is largely substantiated by the results of this study. This is in keeping with our previous finding that patients presenting via open access endoscopy have a more favorable outcome [27]. This further reinforces the importance of early diagnosis. The symptom of new-onset dyspepsia clearly merits a higher public profile. Health education measures are required, not only in the community to educate the public, but also in primary care, to remind general practitioners that gastric cancer presenting with new-onset dyspepsia is potentially curable. Finally, this message needs to be assimilated by health planners when commissioning cancer services, so that geographical areas in the United Kingdom with a high prevalence of esophagogastric cancer are well served by an efficient and well-resourced local cancer network.

Acknowledgments Mr. Blackshaw and Mr. Barry were supported in part by a grant from the ASTRA foundation.

\section{References}

1. CRC (Cancer Research Campaign). Factsheet 1.1 Incidence-UK. London: Cancer Research Campaign; 1998.

2. CRC (Cancer Research Campaign). Cancer Stats Mortality-UK. London: Cancer Research Campaign; 1999.

3. Martin IG, Young S, Sue Ling H, Johnson D. Delays in the diagnosis of oesophagogastric cancer: a consecutive case series. BMJ 1997;314:467-70. 
4. Davies DL, Hoel D, Fox J, Lopez A. International trends in cancer mortality in France, West Germany, Italy, Japan, England and Wales and the USA. Lancet 1990;336:474-81.

5. Mella J, Biffin A, Radcliffe AG. A population-based audit of colorectal cancer management in two United Kingdom health districts. Br J Surg 1997;84:1731-6.

6. Scholefield JH, Robinson MHE, Mangham C. Screening for colorectal cancer reduces emergency admissions. Eur J Surg Oncol 1998;24:47-50.

7. Pye JK, Crumplim MK, Charles J, Kerwat R, Foster ME, Biffin A. One-year survey of carcinoma of the oesophagus and stomach in Wales. Br J Surg 2001;88:278-85.

8. Gilbert FJ, Park KGM, Thompson AM, editors. Scottish Audit of Gastric and Oesophageal Cancer-Report 1997-2000 [online]. http://www.show.scot.nhs.uk/crag/. 2002. [accessed November 21, 2003].

9. Welsh Index of Multiple Deprivation [online]. http:// www.wales.gov.uk/keypubstatisticsforwales/content/publications/ social/2000/deprivation/index_dataxls. 2000. [accessed January 14, 2004].

10. Sue-Ling HM, Johnson D, Martin IG, Dixon MF, Lansdown MRJ, McMahon MJ, et al. Gastric cancer: a curable disease in Britain. BMJ 1993;307:591-6.

11. Lewis WG, Edwards P, Barry JD, Khan S, Dhariwal D, Allison $\mathrm{MC}$, et al. D2 or not D2? The gastrectomy question. Gastric Cancer 2002;5:29-34.

12. Kennedy BJ. The unified international gastric cancer staging classification. Scand J Gastroenterol 1987;22(Suppl 133):11-3.

13. Sobin $\mathrm{LH}$, Wittekind $\mathrm{CH}$, editors. UICC TNM classification of malignant tumours. Fifth Ed. New York: John Wiley and Sons; 1997.

14. Altman DG. Practical statistics for medical research. London: Chapman and Hall; 1991.

15. Kaplan EL, Meier P. Non-parametric estimation from incomplete observations. J Am Stat Assoc 1958;58:457-81.

16. Peto R, Pike MC, Armitage P, Breslow NE, Cox DR, Howard SV, et al. Design and analysis of randomised clinical trials requiring prolonged observation of each patient. Analysis and examples. Br J Cancer 1977;35:1-39.

17. Whiting J, Siggurdsson A, Rowlands DC, Hallissey MT, Fielding JWL. The long term results of endoscopic surveillance of premalignant lesions. Gut 2002;50:378-81.

18. Griffin SM, Raimes SA. Proton pump inhibitors may mask early gastric cancer. BMJ 1998;317:1606-7.

19. Hallissey MT, Allum WH, Jewkes AJ, Ellis DJ, Fielding JWL. Early detection of gastric cancer. BMJ 1990;301:315.

20. Bramble MG, Suvakovic Z, Hungin AP. Detection of upper GI cancers in patients taking antisecretory therapy prior to gastroscopy. Gut 2000 April;46:464-7.

21. Suvakovic Z, Bramble MG. Improving the detection rate of early gastric cancer requires more than open access gastroscopy: a 5year study. Gut 1997;41:308-13.

22. Wayman J, Raimes S, Griffin SM, Hayes N. Small study found that four-fifths of delay was due to patients (letter). BMJ 1997; 315:426-7.

23. Furukawa $H$, Imamura $H$, Kodera $Y$. The role of surgery in the current treatment of gastric carcinoma. Gastric Cancer 2002;5: 13-6.

24. Earle CC, Maroun JA. Adjuvant therapy after curative resection for gastric cancer: revisiting a meta-analysis of randomized trials. Proc Am Soc Clin Oncol 1998;17:263a.

25. Allum W, Cunningham D, Weeden S, for the UK NCRI Upper GI Clinical Studies Group. Perioperative chemotherapy in operable gastric and lower oesophageal cancer: randomised controlled trial (the MAGIC trial). Proc Am Soc Clin Oncol 2003;22:A998.

26. Macdonald JS, Smalley SR, Benedetti J, Hundahl SA, Estes NC, Stemmermann GN, et al. Chemoradiotherapy after surgery compared with surgery alone for adenocarcinoma of the stomach and gastroesophageal junction. N Engl J Med 2001;10:725-30.

27. Blackshaw GR, Barry JD, Edwards P, Allison MC, Lewis WG. Open-access gastroscopy is associated with improved outcomes in gastric cancer. Eur J Gastroenterol Hepatol 2003;15:1333-7. 